\title{
Residual Thermodynamics: A Framework for Analysis of Non-Linear Irreversible Processes
}

\author{
M. Gustavsson \\ Hot Disk AB, c/o Chalmers Science Park, Sven Hultins gata 9, SE-41288 Gothenburg, Sweden \\ E-mail: Mattias.Gustavsson@hotdiskinstruments.com
}

\begin{abstract}
For a general irreversible process, incorporating sub-processes, entropy change may be expressed as the vector product of a thermodynamic force- and flow vector. A fundamental hypothesis is introduced, whereby a general irreversible process or sub-process may be categorized as being of a non-residual type, or of a residual type. A nonresidual irreversible process is typically associated with continuous, linear behavior, where true material properties represent model constants, and the principle of local equilibrium is valid. A residual irreversible process is typically associated with discrete-, non-continuous-, and often non-linear behavior, where model constants do not represent generally accepted material properties.
\end{abstract}

Keywords: Process comparison; sub-process, non-linear.

\section{Introduction}

Residual thermodynamics, in the present context ${ }^{1}$, aims at clarifying the relationships between entropy change, thermodynamic forces and flows for a specific class of irreversible processes. By introducing a fundamental hypothesis, a residual thermodynamics framework can be outlined.

Non-residual irreversible processes typically occur in near-equilibrium systems. Irreversible processes governed by linear phenomenological laws (Kondepudi \& Prigogine, 1998), e.g. laminar fluid flows, continuous thermal conduction in solids etc., are arguably of a non-residual type. Model constants in linear phenomenological laws typically incorporate corresponding well-recognized material properties such as kinematic viscosity, thermal conductivity tensor etc. Residual irreversible processes are, by contrast, not connected with any continuum hypothesis or linear phenomenological laws, and hence do not involve any widely-accepted material properties as model coefficients. (In some situations, however, it is possible to analyze processes assuming apparent material properties as model constants.)

The residual thermodynamics framework can be applied for the analysis of discrete interactions at interfaces between two systems (e.g. particle flow and a target surface, generating erosive wear). This analysis may also be applied for discrete self-comparison analysis [e.g. chemical reactions or mechanical attrition (Gustavsson, submitted)].

Residual irreversible processes typically occur in farfrom equilibrium systems, where thermodynamics is nonlinear and where thresholds play an important role. For instance, fluctuations around thresholds can generate a

\footnotetext{
${ }^{1}$ In the literature, "residual thermodynamics" refers to the modeling of differences in thermodynamic states of substances, e.g. real- versus corresponding ideal gas states.
}

dissipative structure (Kondepudi \& Prigogine, 1998). It appears that material properties have little or no influence on the resulting wavelengths of the dissipative structure. Other conditions, such as oscillations in concentrations or other geometrical constraints, are here shown to have a direct influence on the wavelengths. (Prigogine's general observations on far-from equilibrium behavior are also discussed.)

\section{Theory}

\subsection{Preamble}

For a general irreversible process it is always possible to model entropy change or entropy generation ${ }^{2}$ as the product of an intensive thermodynamic force ${ }^{3} \mathbf{F}$ and an extensive thermodynamic flow ${ }^{4} \mathbf{X}, c f$. (Kondepudi \& Prigogine, 1998):

$$
\mathrm{d}_{\mathrm{i}} S=\mathbf{F} \cdot \mathrm{d} \mathbf{X}
$$

A "real" process involving multiple internal irreversible processes, incorporating one or several irreversible subprocesses of interest to study, is here compared to a corresponding "ideal" process that does not incorporate the specific irreversible sub-processes of interest. Comparisons can be made in many different ways. To make an applicable comparison, the critical issue becomes the specific selection

2 For an isolated group of interacting sub-processes included in the vector representation in Eq. (1), the net sum of entropy change equals the entropy generation, i.e. $\mathrm{d} S_{\text {gen }}=\mathrm{d}_{\mathrm{i}} S, c f$. (Kondepudi \& Prigogine, 1998).

3 A thermodynamic force is also referred to as "affinity" or "generalized force conjugate to the flux $\dot{\mathbf{X}}$ " in the literature, $c f$. (Kestin, 1979).

"A thermodynamic flow is also referred to as "flux" in the literature, $c f$. (Kestin, 1979). 
of ideal process. The following fundamental hypothesis is proposed:

It is always possible to construct an ideal process, having the same thermodynamic flow as the corresponding real process.

This fundamental hypothesis is powerful and general. A direct comparison between a real- and ideal process utilizing the $1^{\text {st }}$ law of thermodynamics is always possible, since there does not exist any flow-rate discrepancy between the two compared processes. (It can be noted that the thermodynamic force and flow in Eq. (1) may appear in corresponding tensorial form, on differentiation.)

\subsection{Framework and Statements}

Consider a system (open or closed, with fixed or moving boundaries), with multiple irreversible subprocesses (the number of sub-processes is assumed to be $N)$. Within this system, it is of interest to study one single particular irreversible sub-process, or one clearly specified "group" of sub-processes. To proceed with mathematical simplicity, consider only one single irreversible sub-process of interest to study, labeled as process nr. N. (A group of sub-processes - where cross-correlations may also occur can be analyzed similarly.)

The $2^{\text {nd }}$ law of thermodynamics provides a detailed expression of the entropy change for process $N$. The summation principle of entropy change applied to all subprocesses results in the net entropy change of the respective real and hypothetical (ideal) process:

$$
\begin{aligned}
& \mathrm{d}_{\mathrm{i}} S_{\text {real }}=\sum_{j=1}^{N} \mathrm{~d}_{\mathrm{i}} S_{j} \\
& \mathrm{~d}_{\mathrm{i}} S_{\text {hyp }}=\sum_{j=1}^{N-1} \mathrm{~d}_{\mathrm{i}} S_{j}
\end{aligned}
$$

Utilizing Eq. (1) for each irreversible sub-process $j$, the entropy change for sub-process $j$ can be formulated as:

$$
\mathrm{d}_{\mathrm{i}} S_{j}=\mathbf{F}_{j} \cdot \mathrm{d} \mathbf{X}_{j}
$$

and Eqs. (2) - (3) can be expressed as:

$$
\begin{aligned}
& \mathrm{d}_{\mathrm{i}} S_{\text {real }}=\sum_{j=1}^{N} \mathbf{F}_{j} \cdot \mathrm{d} \mathbf{X}_{j}=\mathbf{F}_{\text {proc }} \cdot \mathrm{d} \mathbf{Y} \\
& \mathrm{d}_{\mathrm{i}} S_{\text {hyp }}=\sum_{j=1}^{N-1} \mathbf{F}_{j} \cdot \mathrm{d} \mathbf{X}_{j}=\mathbf{F}_{\text {noproc }} \cdot \mathrm{d} \mathbf{Y}
\end{aligned}
$$

The fundamental hypothesis has been applied in Eqs. (5) (6) to obtain the same thermodynamic-flow vector $\mathbf{Y}$. Hence, the residual $2^{\text {nd }}$-law expression for irreversible subprocess $N$ amounts to:

$$
\mathrm{d}_{\mathrm{i}} S_{N}=\mathrm{d}_{\mathrm{i}} S_{\text {real }}-\mathrm{d}_{\mathrm{i}} S_{\text {hyp }}=\left(\mathbf{F}_{\text {proc }}-\mathbf{F}_{\text {noproc }}\right) \cdot \mathrm{d} \mathbf{Y}
$$

It should be noted that Eq. (7a) can often be mathematically simplified into:

$$
\mathrm{d}_{\mathrm{i}} S_{N}=\left(\mathbf{F}_{\text {proc }}-\mathbf{F}_{\text {noproc }}\right) \cdot \mathrm{d} \mathbf{Y}=\left(\mathbf{G}_{\text {proc }}-\mathbf{G}_{\text {noproc }}\right) \cdot \mathrm{d} \mathbf{X}
$$

where vectors $\mathbf{X}$ and $\mathbf{G}$ have lower dimensions than vectors $\mathbf{Y}$ and $\mathbf{F}$.

There are fundamental differences in behavior of subprocess $N$, depending on whether $\mathbf{G}_{\text {noproc }}$ is a zero- or a non-zero vector. The simplification of reducing dimensions in $\mathbf{F}$ and $\mathbf{Y}$ to $\mathbf{G}$ and $\mathbf{X}$ can also be performed through approximation: when performing a socalled continuum approximation or continuum assumption of irreversible process $N$, vectors $\left(\mathbf{F}_{\text {proc }}-\mathbf{F}_{\text {noproc }}\right)$ and $\mathbf{Y}$ (with $\mathbf{F}_{\text {noproc }} \neq \mathbf{0}$ ) are approximated with a corresponding apparent continuous process represented by vectors $\left(\mathbf{G}_{\text {proc }}-\mathbf{G}_{\text {noproc }}\right)$ and $\mathbf{X}$, with $\mathbf{G}_{\text {noproc }}=\mathbf{0}, c f$. Appendix (apparent thermal conductivity (Gustafsson, 1991; Gustavsson, Karawacki \& Gustafsson, 1994; Suleiman, Gustavsson, Karawacki \& Lundén, 1997; Gustavsson \& Gustafsson, 2001). The special case of $\mathbf{G}_{\text {noproc }} \equiv \mathbf{0}$ implies that a so-called linear phenomenological relation ( $c f$. (Kondepudi \& Prigogine, 1998)) can be established, for which it is often possible to assign a material property (or apparent material property) to the process. (Note: If $\mathbf{G}_{\text {noproc }} \neq \mathbf{0}$, then sub-process $N$ is often non-linear, $c f$. discussion following Eq. (10).)

The nature of an irreversible sub-process in terms of Eq. (7b) has five important consequences: First, (C.1) the general model of an irreversible sub-process (Eq. (7b)) also applies for any irreversible process, i.e. Eq. (4a) can more generally be presented as:

$\mathrm{d}_{\mathrm{i}} S_{j}=\left(\mathbf{F}_{\text {proc }, j}-\mathbf{F}_{\text {noproc }, j}\right) \cdot \mathrm{d} \mathbf{X}_{j}$

where summation of all sub-processes in a system provides the total entropy change:

$\mathrm{d}_{\mathrm{i}} S_{\text {tot }}=\sum_{j} \mathrm{~d}_{\mathrm{i}} S_{j}=\sum_{j}\left(\mathbf{F}_{\text {proc }, j}-\mathbf{F}_{\text {noproc }, j}\right) \cdot \mathrm{d} \mathbf{X}_{j}$

Secondly, (C.2) by recursion, re-deriving Eqs. (4a)-(7b) and utilizing the more general form established in Eq. (4b) as an initial assumption, will yield the same result in Eq. (7b). Thirdly, (C.3) any irreversible sub-process $\mathrm{d}_{\mathrm{i}} S_{N}=\left(\mathbf{G}_{\text {proc }}-\mathbf{G}_{\text {noproc }}\right) \cdot \mathrm{d} \mathbf{X}$ with $\mathbf{G}_{\text {noproc }} \neq \mathbf{0}$ can always be considered as an interaction between two discrete thermodynamic systems. Such a sub-process is here referred to as a residual thermodynamic process, where the associated thermodynamic force $\left(\mathbf{G}_{\text {proc }}-\mathbf{G}_{\text {noproc }}\right)$ (with $\mathbf{G}_{\text {noproc }} \neq \mathbf{0}$ ) is in the following referred to as an effective thermodynamic force. The fourth consequence (C.4) stems from an observation stated in the literature ( $c f$. (Kondepudi \& Prigogine, 1998)): when the thermodynamic flow tends toward zero, this always results in the thermodynamic force tending toward zero, and the entropy generation tending toward zero as well. This is generally correct for continuous (non-residual) processes. For residual processes, however, this view needs to be clarified: For instance, consider the (residual) process of discrete heat transfer, e.g. a convection heat transfer process (typically modeled as a discrete interaction between a solid surface and a fluid flow) or a discrete radiation heat transfer process between two thermal 
reservoirs at different temperatures, $T_{1}$ and $T_{2}\left(>T_{1}\right)$, respectively. In both of these cases, the effective thermodynamic force can be expressed in terms of reservoir temperatures according to $F_{\text {proc }}-F_{\text {noproc }}=T_{1}^{-1}-T_{2}^{-1}, c f$. (Kondepudi \& Prigogine, 1998) (p.93). (The thermodynamic flow rate is modeled as

$\dot{Y}=\dot{q} A=\alpha A\left(T_{2}-T_{1}\right)$ (convection)

$\dot{Y}=\dot{q} A=\varepsilon_{s} \sigma A\left(T_{2}^{4}-T_{1}^{4}\right)$ (radiation).)

Gradually

decreasing the interaction between these two thermal reservoirs - for instance by separating them in space for the radiation heat transfer process or reducing the fluid flow rate for the convection process - corresponds to decreasing the thermodynamic flow rate (toward zero) and decreasing the (residual) entropy generation rate (toward zero). But the effective thermodynamic force remains unchanged. Another case where the effective thermodynamic force appears to remain essentially unchanged is the process of flow involving kinetic friction, a discrete interaction between two solid surfaces (and hence a residual process), where the effective thermodynamic force between the two discretely interacting surfaces can be expressed as $F_{\text {proc }}-F_{\text {noproc }}=\mu F_{N} / T$ utilizing the approximate Coulomb friction model. (The Coulomb model does not suggest any specific value of $F_{\text {noproc }} \neq 0$.) Again, according to the Coulomb model, the entropy generation rate will decrease (toward zero) when the thermodynamic flow rate (relative velocity $U$ ) decreases (toward zero), but the effective thermodynamic force will not change 5 . Hence, for residual processes, it is not possible with presently available experimental results to make any firm statements on the general behavior of effective thermodynamic forces when the thermodynamic flow tends toward zero: For a residual process, it is only possible to state that if $\dot{\mathbf{X}} \rightarrow \mathbf{0}$ (or $\dot{\mathbf{Y}} \rightarrow \mathbf{0}$ ), then $\mathrm{d}_{\mathrm{i}} S_{N} / \mathrm{d} t \rightarrow 0$. The fifth and final consequence (C.5) is that discrete/non-continuum irreversible interaction can for some situations be analyzed at sub-continuum scales as an irreversible residual process, $c f$. Section 4.

The following statements summarize the fundamental process differences:

If $\mathbf{F}_{\text {noproc, } j} \equiv \mathbf{0}$, then:

(S.a) A thermodynamic material property or a linear phenomenological coefficient can be associated with the irreversible sub-process $j$ driven by the thermodynamic force $\mathbf{F}_{\text {proc } j}$.

(S.b) The sub-process $\mathrm{d}_{\mathrm{i}} S_{j}=\mathbf{F}_{\text {proc }, j} \cdot \mathrm{d} \mathbf{X}_{j}$ does not represent a residual process.

(S.c) The principle of local equilibrium is valid.

(S.d) Non-residual thermodynamics require (many-particle) averaged behavior (continuum assumption).

\footnotetext{
${ }^{5}$ Regarding friction processes, the author believes that more experimental work is necessary to assess the validity of the Coulomb model at near-zero velocities, $c f$. Figure 1 (left).
}

(S.e) If $\dot{\mathbf{X}}_{j} \rightarrow \mathbf{0}$ then $\mathbf{F}_{\text {proc }, j} \rightarrow \mathbf{0}$ and $\mathrm{d}_{\mathrm{i}} S_{j} / \mathrm{d} t \rightarrow 0$.

If $\mathbf{F}_{\text {noproc, } j} \neq \mathbf{0}$, then:

(S.f) A thermodynamic material property cannot be associated with the irreversible sub-process $j$ driven by the effective thermodynamic force $\left(\mathbf{F}_{\text {proc }, j}-\mathbf{F}_{\text {noproc }, j}\right)$.

(S.g) The sub-process $\quad \mathrm{d}_{\mathrm{i}} S_{j}=\left(\mathbf{F}_{\text {proc }, j}-\mathbf{F}_{\text {noproc }, j}\right) \cdot \mathrm{d} \mathbf{X}_{j}$ represents a residual process.

(S.h) The principle of local equilibrium is not valid.

(S.i) Discrete residual thermodynamic interactions can in some cases also be applied on the local or particle scale (i.e. sub-continuum scales, yet still requiring collective behavior of many particles).

(S.j) The residual process $\mathrm{d}_{\mathrm{i}} S_{j}=\left(\mathbf{F}_{\text {proc }, j}-\mathbf{F}_{\text {noproc }, j}\right) \cdot \mathrm{d} \mathbf{X}_{j}$ can be modeled as a discrete interaction between two systems, either as an interaction between two spatially separated systems, or through a discrete "self comparison".

(S.k) If $\dot{\mathbf{X}}_{j} \rightarrow \mathbf{0}$, then $\mathrm{d}_{\mathrm{i}} S_{j} / \mathrm{d} t \rightarrow 0$. (The effective thermodynamic force $\left(\mathbf{F}_{\text {proc }, j}-\mathbf{F}_{\text {noproc }, j}\right)$ does not necessarily tend to zero.)

The concept of a "thermodynamic" material property is here introduced, to prudently avoid discussions whether some coefficients - such as the emissivity coefficient $\varepsilon_{s}$, the heat transfer coefficient $\alpha$ or the kinetic friction coefficient $\mu$-represent true material properties or not. (The author does not consider these coefficients to be true material properties.)

Turning our attention to the issue of thresholds, consider next an irreversible process $(N=1)$ as a function of flow rate $\dot{\mathbf{X}}_{1}$, where $\mathbf{F}_{\text {noproc, } 1} \equiv \mathbf{0}$. When increasing the flow to a certain rate $\dot{\mathbf{X}}_{1, \text { threshold }}$, a residual process may suddenly initiate, providing an additional entropy change rate $\mathrm{d}_{\mathrm{i}} S_{2} / \mathrm{d} t=\left(\mathbf{F}_{\text {proc }, 2}-\mathbf{F}_{\text {noproc }, 2}\right) \cdot \dot{\mathbf{X}}_{2}$ or entropy generation rate $\quad \mathrm{d} S_{\text {gen }, 2} / \mathrm{d} t=\left(\mathbf{F}_{\text {proc }, 2}-\mathbf{F}_{\text {noproc }, 2}\right) \cdot \dot{\mathbf{X}}_{2}, \quad$ where $\mathbf{F}_{\text {noproc }, 2} \neq \mathbf{0}$. The new residual process might, according to the above, require extended dimensions of the thermodynamic flow rate and thermodynamic forces (which were unforeseen or excluded when modeling the entropy generation rate at sub-threshold levels of the flow rate). If so, it is possible to re-model sub-threshold levels with corresponding zero terms in extended vectors, i.e. $\mathrm{d}_{\mathrm{i}} S_{\text {res }} / \mathrm{d} t\left(=\mathrm{d}_{\mathrm{i}} S_{2} / \mathrm{d} t\right)$ or $\mathrm{d} S_{\mathrm{gen}, \text { res }} / \mathrm{d} t\left(=\mathrm{dS}_{\mathrm{gen}, 2} / \mathrm{d} t\right)$ equal to $\sum_{j=1}^{2}\left(\mathbf{F}_{\text {proc }, j}-\mathbf{F}_{\text {noproc }, j}\right) \cdot \dot{\mathbf{X}}_{j}=\left(\mathbf{F}_{\text {proc }}-\mathbf{F}_{\text {noproc }}\right) \cdot \dot{\mathbf{Y}}$. The residual process can appear as a distinct threshold process, i.e. providing a non-linear behavior according to Figure 1 (right).

An important practical illustration regarding thresholds is the case of erosion, which can be analyzed as an irreversible residual process, $c f$. (Gustavsson, submitted). It is well known from single-particle erosion experiments that erosive wear will not occur for a particle having a low 


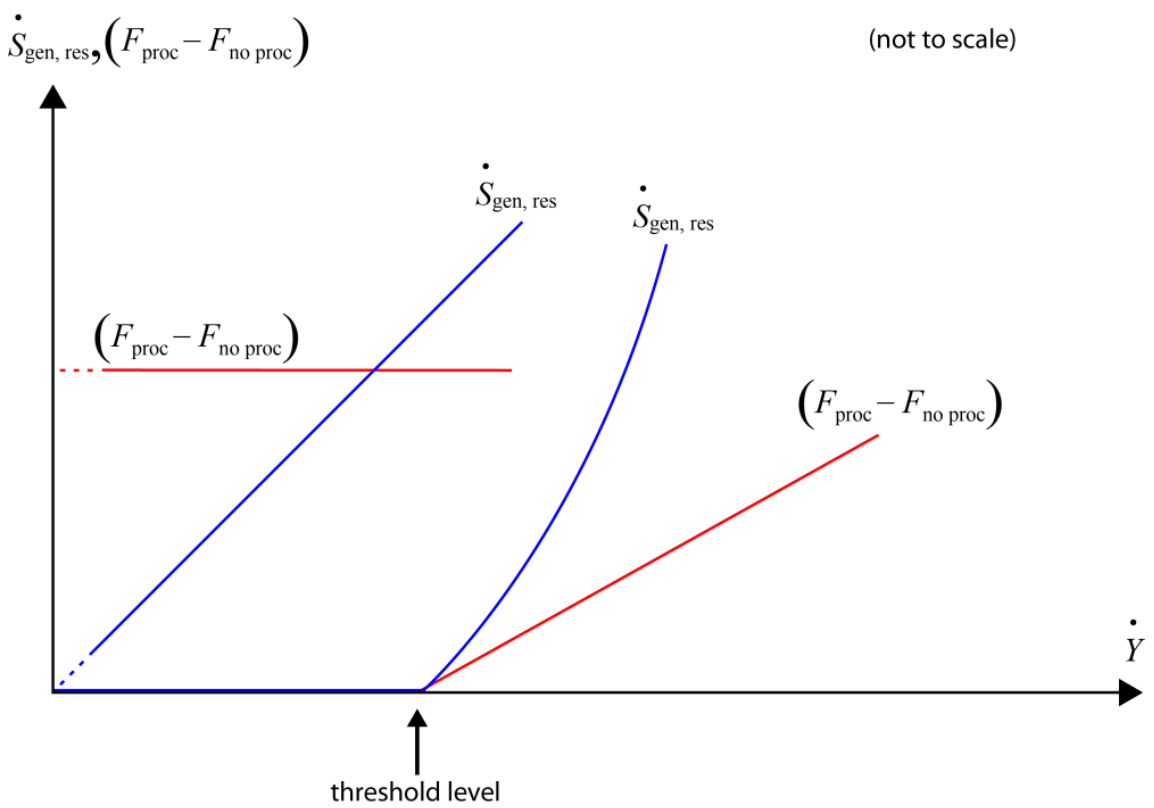

Figure 1. Schematic 1D illustration of thermodynamic flows, thermodynamic forces and threshold level for two different (isolated) residual processes.

relative impact velocity. Exceeding a certain threshold velocity, however, erosive wear will commence. ${ }^{6}$

\section{Interpretations and Applications \\ 3.1 Discrete Interaction and Self Comparisons}

The extant literature presents several examples of irreversible interaction between two discrete systems, e.g.: (a) expansion of a volume defined by a piston due to an internal pressure $P_{\mathrm{gas}}$, for a piston at pressure $P_{\text {piston }}$ : $\mathrm{d}_{\mathrm{i}} S / \mathrm{d} t=\left(\left(P_{\text {gas }}-P_{\text {piston }}\right) / T\right)(\mathrm{d} V / \mathrm{d} t),($ b) diffusion between two discrete systems driven by chemical potential differences: $\quad \mathrm{d}_{\mathrm{i}} S / \mathrm{d} t=\left(-\left(\mu_{2}-\mu_{1}\right) / T\right)(\mathrm{d} \xi / \mathrm{d} t)$, where $\mu_{\mathrm{i}}$ represents the chemical potential, and $\xi$ represents the extent of reaction. These situations are traditionally analyzed as monitoring the net sum of entropy change for two geometrically separated discrete systems, providing the entropy change for the entire process.

Alternatively, self-comparisons can be performed by utilizing the fundamental hypothesis: For case (a), comparing the expansion due to a (process) pressure $P_{\text {gas }}$ with an identical expansion at (no-process) pressure $P_{\text {piston }}$, gives the same result with $\left(F_{\text {proc }}-F_{\text {noproc }}\right)=\left(P_{\text {gas }}-P_{\text {piston }}\right) / T$ and $\dot{Y}=\mathrm{d} V / \mathrm{d} t$. The fundamental hypothesis may always be applied, regardless

${ }^{6}$ In the field of Wear, erosion experiments are often conducted for single-particle impact in vacuum at impacting speeds far beyond the erosion threshold, typically of the order of $100 \mathrm{~m} / \mathrm{s}$ or similar; by experimentally suppressing the threshold influence in this way to a negligible relative influence, the ensuing principal erosion behavior is much more easily analyzed, and erosion models based on Newtonian analysis can be successfully derived. of whether the process is a residual thermodynamic process or not. In this case $F_{\text {noproc }}=P_{\text {piston }} / T$, hence case (a) represents an irreversible residual thermodynamic process. An additional outcome for case (a) is that, according to statement (S.f), an irreversible piston expansion driven by a discrete pressure difference cannot be associated with a thermodynamic material property. Case (b) can be analyzed similarly as a chemical reaction, $c f$. Appendix (chemical reactions). The difference would be, according to (Kondepudi \& Prigogine, 1998) (p.114), that one would associate the thermodynamic flow with an "extent of reaction", since no true chemical reaction would be occurring.

Self-comparison also applies for corresponding $1^{\text {st }}$-law expressions. For instance, consider the flow work of surface forces in a multiphase flow (Panton, 1984; Ishii, 1975) ${ }^{7}$. A differential-scale self-comparison of the product between the general force tensor $\stackrel{\bar{T}}{T}=-p \overline{\bar{I}}+\bar{\tau}$ and the flow velocity vector can be stated (for each individual fluid phase) as: $-\nabla \cdot\left(\alpha\left(\overline{=}_{\text {proc }}-\overline{\bar{T}}_{\text {noproc }}\right) \cdot \mathbf{U}\right)=$

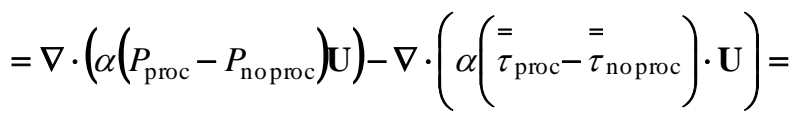

$=\underbrace{\alpha\left(P_{\text {proc }}-P_{\text {noproc }}\right) \nabla \cdot \mathbf{U}}+\underbrace{\mathbf{U} \cdot \nabla\left(\alpha\left(P_{\text {proc }}-P_{\text {noproc }}\right)\right)}$

(a) $(\mathrm{b})$

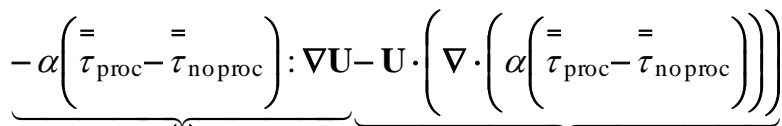

(c)

(d)

\footnotetext{
${ }^{7}$ Regarding fluctuations or turbulence, $c f$. Section 4.
} 
This expression can be used to obtain some earlier and well-known results in fluid dynamics, $c f$. Appendix (Newtonian fluid dynamics). Also, Eq. (9) makes it possible to derive new residual thermodynamic expressions, for instance general wear and attrition mechanisms, $c f$. (Gustavsson, submitted).

\subsection{Apparent Thermodynamic Material Properties}

For irreversible processes incorporating at least one irreversible residual sub-process - according to statement (S.f) - one cannot, strictly speaking, assign a true thermodynamic material property as phenomenological model factor to the net irreversible process in Eq. (8). However, for situations where irreversible residual subprocesses apply - for instance at a limited range of operating conditions and avoiding process thresholds - one may approximate the process by assuming apparent thermodynamic material properties.

Consider the experimental procedure for the Vickers- or Brinell hardness tests ${ }^{8}$ : The net irreversible indentation (due to work hardening and/or fracture) of a pyramid-shaped tip into a target material, rendering the Vickers hardness number, can be considered as the net space-time integrated result of the following three processes (which may occur in geometrically separable zones within the deformed target material), together with other simultaneously occurring processes such as friction:

1. Indentation in a fully elastic zone - conceptually similar to indentation of a rubber-type material.

2. Indentation in an elastic-plastic zone.

3. Indentation in a purely plastic zone.

Categorizing these three sub-processes for the net process irreversible indentation, case (1) gives $\left(\mathbf{F}_{\text {proc }}-\mathbf{F}_{\text {noproc }}\right)=\mathbf{0}$, case (2) gives $\left(\mathbf{F}_{\text {proc }}-\mathbf{F}_{\text {noproc }}\right)>\mathbf{0}$ where $\mathbf{F}_{\text {noproc }} \neq \mathbf{0}$, and case (3) gives $\left(\mathbf{F}_{\text {proc }}-\mathbf{F}_{\text {noproc }}\right)>\mathbf{0}$ where $\mathbf{F}_{\text {noproc }}=\mathbf{0}$.

It directly follows that it is not possible to determine a universal hardness number. This is evident from the number of different experimental procedures for hardness testing, which all produce different hardness numbers for the same tested material.

\section{Residual Process Work-Loss-Induced Fluctuations}

In (Kondepudi \& Prigogine, 1998) the concept of structural instability is discussed; when adding a chemical reaction to a series of chemical reactions, the net result will often be that of new cross-chemical reactions and significantly increased entropy generation for the entire system. (Adding chemical reactions to a series of chemical reactions will typically significantly increase chemical affinities involved (Kondepudi \& Prigogine, 1998).) Also, new "states" or ("dissipative") "structures" of processes within the system may emerge as a result of adding a chemical reaction. These changes correspond to extending the dimensions and adjusting (typically increasing) the magnitudes of effective thermodynamic force- and flow vector components in Eq. (1).

\footnotetext{
${ }^{8}$ There exist multiple types of tests and measures of hardness, which indicates that this concept may not be so well defined.
}

From a mechanical engineer's point of view, to provoke a corresponding instable process in mechanics or fluid dynamics by simply "adding" processes is hard to conceive. On the other hand, from a thermodynamics point of view, a certain degree of corresponding behavior in mechanical systems might occur with significantly increased thermodynamic flow rates or thermodynamic forces.

Consider Prigogine's general observations (for chemical systems):

- (P.1) Structural instability in chemical systems is typically connected with so-called "thresholds" and increased entropy generation, $c f$. Figure 19.13 in (Kondepudi \& Prigogine, 1998). ${ }^{9}$

- (P.2) At far-from-equilibrium conditions, "linear phenomenological relations are no longer valid".

- (P.3) At far-from-equilibrium, regular structures are generated, referred to as "dissipative structures", which are "typically of very complex behavior, such as for instance oscillations in concentration, propagating waves, and chaos".

- (P.4) At far-from-equilibrium conditions, the "state is preserved through a continual flow of energy or matter, or both".

The literature discusses several different models and examples, where for instance numerical simulation of farfrom-equilibrium conditions, instability and other nonlinear situations can reproduce experimental behavior fairly well, on a case-to-case basis. Hitherto presented models for non-linear irreversible thermodynamic processes, however, do not share common theoretical ground ${ }^{10}$. The absence of a theory that connects Prigogine's important observations (P.1)-(P.4) to thermodynamics on a fundamental level makes it interesting to consider these observations within the context of the present residual thermodynamics framework.

Far-from-equilibrium processes arguably fall within the classification of residual thermodynamic processes. Observation (P.2) has a corresponding statement (S.f) - no thermodynamic material property can be assigned as model constant in a far-from-equilibrium process. In addition, observation (P.4) is a basic precondition for any residual thermodynamic process. Observation (P.1) fits within the residual thermodynamics framework, where process thresholds and increased entropy generation are intimately related to residual thermodynamic processes, $c f$. Section 2 and Figure 1. The remaining challenge and focus of this section is to relate and provide an analysis of Prigogine's observation (P.3) within the residual thermodynamics framework.

To simplify, consider residual processes of type:

$$
\dot{S}_{\text {gen }, \text { res }}=\left(\mathbf{F}_{\text {proc }}-\mathbf{F}_{\text {noproc }}\right) \cdot \dot{\mathbf{Y}} \geq 0
$$

9 The difference in behavior for structurally instable systems as compared to processes occurring near equilibrium is discussed in (Kondepudi \& Prigogine, 1998) and elsewhere.

${ }^{10}$ In (Kestin, 1979), it was concluded that no generallyaccepted theory of non-equilibrium thermodynamics has yet been developed. 
A residual thermodynamic process, starting to occur at a threshold flow as in Figure 1 (right), by definition represents a non-linear condition. A non-linear regime is defined in the literature as one where the thermodynamic flow is no longer a linear function of the thermodynamic force. It immediately becomes clear, for instance, by comparing the behavior of effective thermodynamic forces at flows arbitrarily below or above the threshold flow at position $\mathrm{B}$ in Figure 2, that no consistent linearity can be established.

To simplify the mathematics, assume a 1D-situation according to Figure 2. Consider the flow condition B,

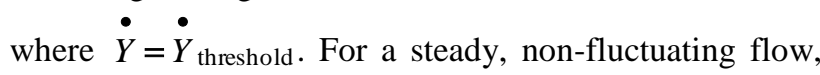
the time-averaged residual entropy generation is zero, i.e. $\overline{S_{\text {gen,es }}}=0$. Now assume that a flow disturbance of the form $\quad \dot{Y}=\dot{Y}_{\text {threshold }}+\dot{Y}_{\mathrm{dev}}$ is introduced, where $\dot{Y}_{\mathrm{dev}}=A \sin \omega t$ and $A$ is an arbitrary real number. It is clear that if $\dot{Y}_{\mathrm{dev}}>0$ then $\left(F_{\text {proc }}-F_{\text {noproc }}\right)=K \dot{Y}_{\mathrm{dev}}$, where $K$ represents the slope, and when $\dot{Y}_{\mathrm{dev}} \leq 0$ then $\left(F_{\text {proc }}-F_{\text {noproc }}\right)=0$. A simple integration, for a full cycle, provides an increased time-averaged entropy generation $\dot{S}_{\text {gen,res,fluct }}=A K^{2} / 4$, due to the flow fluctuations. Interestingly, the increased entropy generation in this case (for a work-loss induced fluctuation) does not depend on any specific frequency, but there is a dependency on primarily the effective thermodynamic force as well as on the amplitude of the fluctuations. A dissipative structure will follow as a result of the on/off residual process, with a pattern structure of wavelength $\lambda=2 \pi \dot{Y}_{\text {threshold }} / \omega$. For mechanical systems subject to work-loss-induced fluctuations, it appears that geometrical conditions have a dominating impact on the resulting dissipative structure wavelength, $c f$. Appendix (erosion ripple phenomenon (Finnie \& Kabil, 1965; Chapman \& Cowling, 1991; Ding, Lyczkowski, Burge, Gidaspow, 1992; Kuuni \& Levenspiel, 1991; Gustavsson, 2002), length of paper fibers produced in a TMP wood chip refiner (Berg, Karlström \& Gustavsson, 2003; Karlström, Sikter \& Gustavsson, 2007; Karlström, Eriksson, Sikter \& Gustavsson, 2008)).

Additional fluctuation analysis (in both thermodynamic flows and effective thermodynamic forces) may be performed in a similar way at the various positions A-D in Figure 2, from which one may conclude that dissipative structures may emerge through three different mechanisms:

- Dissipative structures obtained for residual processes through fluctuations in the flow.

- Dissipative structures obtained for residual processes through fluctuations in the effective thermodynamic forces.

- Dissipative structures obtained for residual processes through dual-coupled (cross-correlation) fluctuations in both thermodynamic flow and effective thermodynamic forces.

Table 1 summarizes the influence on time-averaged residual entropy generation for these three different mechanisms, acting under principally different circumstances. Regular structures, as described by Prigogine's observations in (P.3), appear to form in situations where either residual entropy is generated due to fluctuations, or where time-averaged entropy generation is increased, due to fluctuations. Different behaviors can be observed:

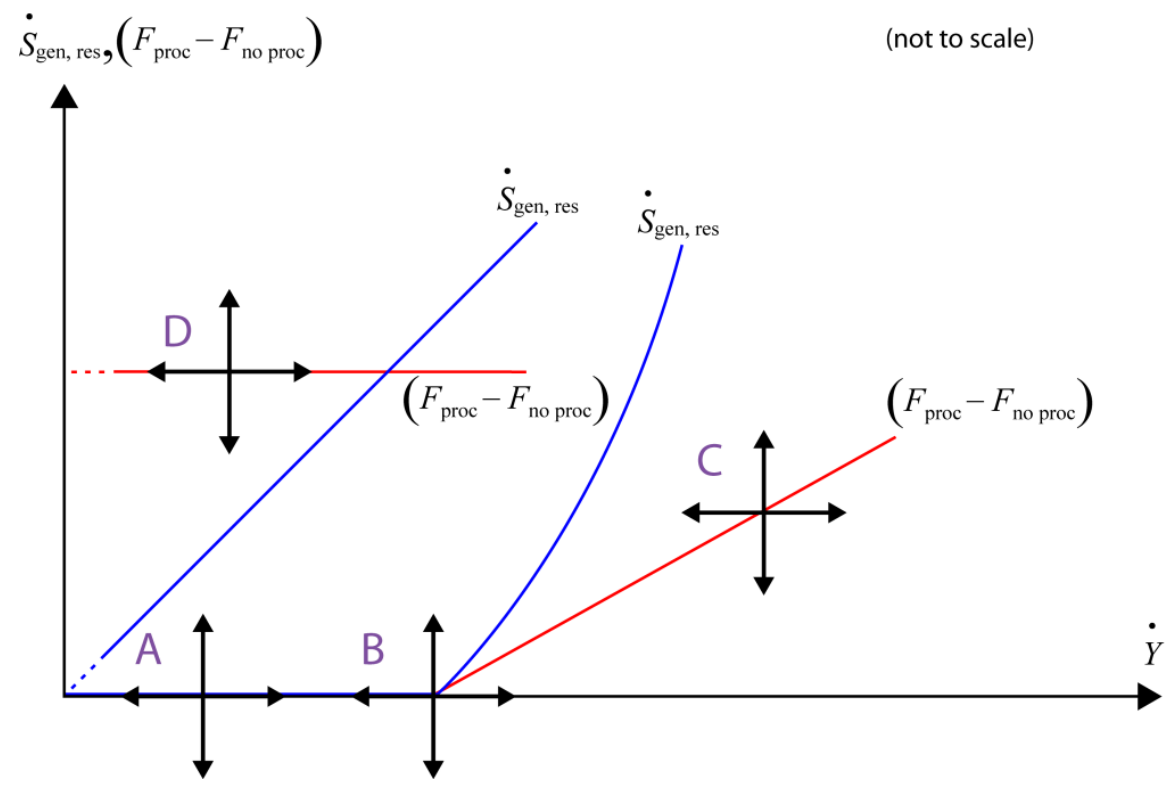

Figure 2. Schematic 1D illustration of effective thermodynamic forces and residual entropy generation for two different (isolated) residual processes, as a function of the thermodynamic flow. Position B represents a threshold (flow). 
Table I. Schematic influence of fluctuations in the vicinity of positions A-D in Fig. II. If time-averaged entropy generation is positive, dissipative structures may emerge.

\begin{tabular}{|c|c|c|c|c|c|c|}
\hline A & $\begin{array}{l}\stackrel{\text { Th.dyn }}{\text { flow }}\end{array}$ & $\overline{\dot{S}_{\text {genresfluct }}}=0$ & $\begin{array}{l}\uparrow \\
\text { Eff. } \\
\text { Th.dyn. } \\
\text { forces }\end{array}$ & $\overline{\dot{S}_{\text {genresfluct }}}>0$ & $\begin{array}{l}\text { Dual } \\
\text { coupled }\end{array}$ & $\overline{\dot{S}_{\text {gentesfluct }}}>0$ \\
\hline B & $\begin{array}{l}\stackrel{\longleftrightarrow}{\text { Th.dyn }} \\
\text { flow }\end{array}$ & $\overline{\dot{S}_{\text {genresfluct }}}>0$ & $\begin{array}{l}\uparrow \\
\text { Eff. } \\
\text { Th.dyn. } \\
\text { forces }\end{array}$ & $\overline{\overline{S_{\text {genresfluct }}}}>0$ & $\begin{array}{l}\text { Dual } \\
\text { coupled }\end{array}$ & $\overline{\overline{\dot{S}_{\text {genresfluct }}}}>0$ \\
\hline $\mathrm{C}$ & $\begin{array}{l}\stackrel{\leftrightarrow}{\text { Th.dyn }} \\
\text { flow }\end{array}$ & $\overline{\overline{S_{\text {genres,fluct }}}}>0$ & $\begin{array}{l}\uparrow \\
\text { Eff. } \\
\text { Th.dyn. } \\
\text { forces }\end{array}$ & $\overline{\dot{S}_{\text {genres,fluct }}} \geq 0$ & $\begin{array}{l}\nearrow \\
\text { Dual } \\
\text { coupled }\end{array}$ & $\overline{\dot{S}_{\text {genres,fluct }}}>0$ \\
\hline $\bar{D}$ & $\begin{array}{l}\stackrel{\leftrightarrow}{\text { Th.dyn }} \\
\text { flow }\end{array}$ & $\overline{\dot{S}_{\text {genres }, \text { fluct }}}=0$ & $\begin{array}{l}\uparrow \\
\text { Eff. } \\
\text { Th.dyn. } \\
\text { forces }\end{array}$ & $\overline{\dot{S}_{\text {genres,fluct }}} \geq 0$ & $\begin{array}{l}\nearrow \\
\text { Dual } \\
\text { coupled }\end{array}$ & $\overline{\dot{S}_{\text {genres,fluct }}}>0$ \\
\hline
\end{tabular}

Flow fluctuations for position A and D in Figure 2 will neither render any additional time-averaged work loss, nor any time-averaged increase in entropy generation. However, fluctuations in the effective thermodynamic forces will render residual entropy generation for position A, where dissipative structures may appear as more chaotic and strong fluctuations in concentration can be induced, e.g. the brittle attrition process in a TMP wood chip refiner, $c f$. Appendix (length of paper fibers produced in a TMP wood chip refiner). This agrees well with Prigogine's observation (P.3). Residual processes of type D, however, when subject to fluctuations in the effective thermodynamic force, typically appear to have little effect on overall net entropy generation, except in the case of dual-coupled fluctuations or possibly if the forces fluctuate in a non-linear manner. For instance, friction or abrasion processes (which can be modeled as category type D processes) are from experimental tests seldom directly associated with flow fluctuations or ripple phenomena, although such phenomena may be observed e.g. at near-zero velocities for friction, or in situations of severe abrasion. Fluctuations at position $\mathrm{B}$ and $\mathrm{C}$, in particular flow fluctuations, will typically generate a dissipative structure of regular wavelength. These dissipative structures have a propagating behavior and may also induce local oscillations in concentration, $c f$. Appendix (erosion ripple phenomenon). This also agrees well with Prigogine's observation (P.3).

\section{Conclusions}

A fundamental hypothesis is introduced, by which a framework of residual thermodynamics is outlined. This new framework is successfully employed to derive basic models of inert wear and attrition mechanisms (Gustavsson, submitted). It provides the necessary tools to analyze discrete and non-linear sub-processes, which in the case of wear and attrition occur on sub-continuum scales. A residual process, e.g. wear and attrition, is typically associated with threshold phenomena. Also, typical for a residual process is that model coefficients do not represent any widely-accepted bulk material properties.

The present framework introduces a distinct split-up of a general irreversible thermodynamic (sub-)process into either a residual-type irreversible (sub-)process or a nonresidual-type irreversible (sub-)process. The formulation of a sub-process in terms of Eq. (4b), when $\mathbf{F}_{\text {noproc }, j} \neq \mathbf{0}$, is mathematically discrete as it either represents a discrete interaction between two systems at an interface, or as a discrete self-comparison.

Regarding the nature of a residual thermodynamic process, it can be observed that the analysis of such a nonlinear process - with the present approach - may render significantly different results as compared to such obtained by alternative thermodynamic approaches (assuming the principle of local equilibrium to be valid). However, such deviations should not be considered contradictory to earlier work in this field, but require some comment: Firstly - and obviously - deviations in results are to be expected, when assumptions regarding the behavior of the original system are assumed to be different. Secondly, if the behavior of the original system is not known and assumptions are made, which in turn lead to satisfactory results, the goal has been achieved.

Kondepudi \& Prigogine (1998) (p. 459) discuss the assumption of the principle of local equilibrium. They state that even if the principle of local equilibrium is not strictly valid, it can often be assumed with good accuracy. This applies specifically when one models processes which incorporate residual thermodynamic sub-processes. Accurate approximations can be made by assuming a continuum process employing apparent thermodynamic 
material properties. For a stationary flow, or for a slowly evolving transient flow, such approximation will often give satisfactory results. However, the picture becomes more complex when one considers sharp non-linear process fluctuations. According to (Kondepudi \& Prigogine, 1998) (p. 459) the principle of local equilibrium assumption necessarily requires an exponential decay of fluctuations, which is significantly faster than often found in related experiments. Kondepudi \& Prigogine (1998) (p. 459) thus assert that so-called memory effects cannot exist if the principle of local equilibrium would be valid within the system (at every location).

One may consider the equivalent converse statement of this assertion: Memory effects are possible to model within the system, if the principle of local equilibrium is not valid at every position within the system. This converse statement is in line with results presented in the present paper: Often, discrete residual processes only occur in a very small fraction of the system (in some cases, e.g. attrition, they may occur in a close-to-zero-volume region). The process of brittle attrition in a TMP refiner (which occurs at only some specific positions) forms traces in the shape of flocculation patterns, $c f$. Appendix. This is an experimental example of memory effects, which cannot be obtained if one assumes the principle of local equilibrium to be valid everywhere in the system. The converse statement can hence be utilized as a practical experimental indicator of the nature of the processes occurring within a system: If slow-decaying fluctuations or memory effects are found in a system, then this implies that a significant residual subprocess is active - somewhere - within the system.

On a practical note, a model based on a residual process may appear straightforward to derive - sometimes with assistance of a constitutive relation, and often a boundary condition analysis. However, while a derived model may itself appear simple in formulation, the relevant thermodynamic flow - required as model input - can be difficult to estimate. For instance, in a fluctuating residual process, both the effective thermodynamic force- and associated thermodynamic flow fluctuations may spread spatially and temporally. Such behavior often leads to a modification in macroscopic overall process behavior, which in turn also often influences boundary conditions, $c f$. e.g. Appendix (erosion ripple process, illustrating a gradual transition). From a computational point-of-view, one may employ iterative computations at each time step - a common approach when numerically solving non-linear ordinary- or partial differential equations. As a further complicating factor, it can be expected that threshold levels inherent in the residual model - which represent the critical conditions of a residual process - as well as incorporated model coefficients, will likely change during a macroscopic-scale transition. These latter adjustments need also be accounted for in the iterations at each computational time step, when modeling. Finally, additional residual subprocesses may be triggered at some point, which will further complicate the overall process behavior, and hence modeling. Correlation of model coefficients with corresponding experiments - when possible - is here believed to be the preferred approach to finding specific coefficients and threshold levels.

\section{Acknowledgement}

This work was supported by Hot Disk AB (Sweden). Special thanks to D.Sc. S.E. Gustafsson at Dept. Physics,
Chalmers Univ. of Technology, Mr. H. Otterberg at University of Gothenburg, and Dr. J. Gustavsson, Dept. Microtechnology \& Nanoscience, Chalmers Univ. of Technology, for assistance in preparing this manuscript.

\section{References}

Berg, D., Karlström, A. \& Gustavsson, M. (2003) International mechanical pulping conference, 361366.

Chapman, S. \& Cowling, T. G. (1991). The Mathematical Theory of Non-uniform Gases; An Account of the Kinetic Theory of Viscosity, Thermal Conduction and Diffusion in Gases, $3^{\text {rd }}$ Ed., Cambridge University Press, Cambridge, New York, U.S.A.

Ding, J., Lyczkowski, R. W., Burge, S. W., Gidaspow, D. (1992). Three-dimensional models of hydrodynamics and erosion in fluidised-bed combustors. AIChE Symp. Ser. 88, 85-98.

Finnie, I. \& Kabil, Y. H. (1965). On the formation of surface ripples during erosion. Wear 8, 60-69.

Gustafsson, S. E. (1991). Transient plane source techniques for thermal conductivity and thermal diffusivity measurements of solid materials. Rev. Sci. Instrum. 62(3), 797-804.

Gustavsson, M., (2002). Fluid dynamic mechanisms of particle flow causing ductile and brittle erosion. Wear 252, 845-858.

Gustavsson, M. A Residual Thermodynamics Analysis of Inert Wear and Attrition. (submitted)

Gustavsson, M., \& Gustafsson, S. E. (2001). On the Use of Transient Plane Source Sensors for Studying Materials with Direction Dependent Properties. Thermal Conductivity 26, DEStech Publications Inc., Lancaster, PA, 367-377.

Gustavsson, M., Karawacki E., \& Gustafsson, S. E. (1994). Thermal conductivity, thermal diffusivity, and specific heat of thin samples from transient measurements with hot disk sensors. Rev. Sci. Instrum. 65(12), 3856-3859.

Kuuni, D. \& Levenspiel, O., (1991). Fluidization Engineering $2^{\text {nd }}$ ed., Butterworth-Heinemann, Stoneham, Massachusetts, U.S.A.

Ishii, M., (1975). Thermo-Fluid Dynamic Theory of TwoPhase Flows, Eyerolles, Paris.

Karlström, A., Sikter, D., \& Gustavsson, M. (2007). Plate gap estimation based on physical refining measurements. International Mechanical Pulping Conference.

Karlström, A., Eriksson, K., Sikter, D. \& Gustavsson, M. (2008). Nord. Pulp Paper Res. 23(1) 129-138.

Kestin, J. (1979). A Course in Thermodynamics, Vol. II. Taylor \& Francis.

Kondepudi, D. \& Prigogine, I. (1998). Modern Thermodynamics: From Heat Engines to Dissipative Structures. Wiley.

Panton, R. L., (1984). Incompressible Flow, John Wiley \& Sons, New York, U.S.A. 
Suleiman, B. M., Gustavsson, M., Karawacki, E. \& Lundén, A. (1997). Thermal properties of lithium sulphate. J. Phys. D: Appl. Phys. 30, 2553-2560.

\section{APPENDIX}

\section{A1. Apparent Thermal Conductivity}

The Hot Disk technique (Gustafsson, 1991; Gustavsson et al., 1994; Suleiman et al., 1997; Gustavsson \& Gustafsson, 2001), employing a thin, embedded doublespiral sensor, is capable of accurately recording the uniaxial anisotropic thermal transport properties of a solid structure in a single experiment. The geometric average value of the thermal conductivity in the radial (in-plane) and axial (through-plane) direction with respect to the circular sensor is recorded, together with the thermal diffusivity in the corresponding radial (in-plane) direction. With an externally known volumetric specific heat value, the thermal conductivities and thermal diffusivities in the in-plane- as well as the through-plane directions can be separated. For the industrially important case of stacklayered samples, the inter-layer thermal contact resistances can be analyzed. It has been experimentally observed that for the Hot Disk technique it is necessary to allow heat to disperse through typically more than 10 layers in the through-direction to stabilize the estimation of apparent thermal conductivity properties for both the in-plane- and through-plane directions of the stack structure in question. This experimental approach makes it possible to perform thermophysical property analysis of electronic structures such as PCBs, Li-Ion battery structures etc., with typical anisotropic ratios of the order of 100:1 to 1000:1. The case of a stack sample structure of smooth $\mathrm{Cu}$ sheets, where heat has been allowed to penetrate more than 10 layers, is shown in Table A1 and A2, cf. (Gustavsson \& Gustafsson, 2001).

The averaged impact of individual interfacial thermal contact resistances between layers corresponds to $R_{\mathrm{t}, \mathrm{c}}=3.42 \cdot 10^{-4} \mathrm{~m}^{2} \mathrm{KW}^{-1}$ at $1.8 \mathrm{kPa}$ pressure and $R_{\mathrm{t}, \mathrm{c}}=2.40 \cdot 10^{-4} \mathrm{~m}^{2} \mathrm{KW}^{-1}$ at $7.5 \mathrm{kPa}$ pressure, $c f$. (Gustavsson, 2001).

Table A1. Piled copper sample of $0.2 \mathrm{~mm}$ thick smooth sheets at $1.8 \mathrm{kPa}$ mounting pressure. Specific heat assumed to be approx. $1.5 \%$ lower than tabulated value of copper due to the presence of air layers. Assumed for the effective bulk composite structure: $\rho c_{p}=3.44 \mathrm{MJm}^{-3} \mathrm{~K}^{-1}, \quad c f$. (Gustavsson \& Gustafsson, 2001).

\begin{tabular}{|ccccc|}
\hline $\begin{array}{c}\text { Meas. } \\
\text { Nr. }\end{array}$ & $\begin{array}{c}\lambda_{\mathrm{r}} \\
{\left[\mathbf{W m}^{-1} \mathbf{K}^{-\mathbf{1}}\right]}\end{array}$ & $\begin{array}{c}\lambda_{\mathrm{a}} \\
{\left[\mathbf{W m}^{-1} \mathbf{K}^{-\mathbf{1}}\right]}\end{array}$ & $\begin{array}{c}a_{\mathrm{r}} \\
{\left[\mathbf{m m}^{2} / \mathbf{s}\right]}\end{array}$ & $\begin{array}{c}a_{\mathrm{a}} \\
{\left[\mathbf{m m}^{\mathbf{2}} / \mathbf{s}\right]}\end{array}$ \\
\hline 1 & 384.6 & 0.5963 & 111.8 & 0.1734 \\
2 & 388.3 & 0.5862 & 112.9 & 0.1704 \\
3 & 389.0 & 0.5884 & 113.1 & 0.1710 \\
4 & 383.7 & 0.5999 & 111.5 & 0.1744 \\
Av. & 386.4 & 0.5927 & 112.3 & 0.1723 \\
\hline
\end{tabular}

Table A2. Piled copper sample of $0.2 \mathrm{~mm}$ thick smooth sheets at $7.5 \mathrm{kPa}$ mounting pressure. Specific heat assumed to be approx. $1.5 \%$ lower than tabulated value of copper due to the presence of air layers. Assumed for the effective bulk composite structure: $\quad \rho c_{p}=3.44 \mathrm{MJm}^{-3} \mathrm{~K}^{-1}, \quad c f$. (Gustavsson \& Gustafsson, 2001).

\begin{tabular}{|ccccc|}
\hline $\begin{array}{c}\text { Meas. } \\
\mathbf{N r} .\end{array}$ & $\begin{array}{c}\lambda_{\mathrm{r}} \\
{\left[\mathbf{W m}^{-1} \mathbf{K}^{-\mathbf{1}}\right]}\end{array}$ & $\begin{array}{c}\lambda_{\mathrm{a}} \\
{\left[\mathbf{W m}^{-\mathbf{1}} \mathbf{K}^{-\mathbf{1}}\right]}\end{array}$ & $\begin{array}{c}a_{\mathrm{r}} \\
{\left[\mathbf{m m}^{\mathbf{2}} / \mathbf{s}\right]}\end{array}$ & $\begin{array}{c}a_{\mathrm{a}} \\
{\left[\mathbf{m m}^{\mathbf{2}} / \mathbf{s}\right]}\end{array}$ \\
\hline 1 & 386.2 & 0.8483 & 112.3 & 0.2466 \\
2 & 390.1 & 0.8328 & 113.4 & 0.2421 \\
3 & 385.5 & 0.8534 & 112.1 & 0.2481 \\
4 & 387.7 & 0.8458 & 112.7 & 0.2459 \\
Av. & 387.4 & 0.8451 & 112.6 & 0.2457 \\
\hline
\end{tabular}

The present stack structures can be modeled in a couple of different ways:

- Considering the stack sample as a continuous, homogeneous anisotropic structure, i.e. with no residual processes, $\quad \mathbf{F}_{\text {noproc, } j} \cong \mathbf{0}$, and with apparent thermophysical properties in accordance with average values in Table A1 and A2. (This apparent anisotropic bulk material property assumption was assumed when analyzing the measurements in (Gustavsson \& Gustafsson, 2001).)

- A one-dimensional stationary heat flow across the layers in accordance with a simplified residual thermodynamic expression: Consider the case "process" = stationary heat flow of $1 \mathrm{~W} / \mathrm{m}^{2}$ across a $1 \mathrm{~m}$ wide stack sample, at $300 \mathrm{~K}$. An accurate determination of the sample sheets reveals that 4926 layers correspond to a $1 \mathrm{~m}$ wide stack sample, each layer corresponding (including some $1.5 \%$ volume air between layers) to an effective thickness of $0.203 \mathrm{~mm}$ (each individual copper layer is $0.200 \mathrm{~mm}$ thick, with an approximate intrinsic thermal conductivity of $392 \mathrm{~W} / \mathrm{mK}$ ). The stack sample at $1.8 \mathrm{kPa}$ mechanical pressure has $G_{\text {proc }}=\left(T_{1 c}{ }^{-1}-T_{1 h}{ }^{-1}\right)=(1 / 300-1 / 301.687)=$ $=1.864 \cdot 10^{-5} \mathrm{~K}^{-1}$. The corresponding ideal process (i.e. identical heat flow without the presence of thermal contact interfaces) has $G_{\text {noproc }}=\left(T_{1 c}^{-1}-T_{1 h}{ }^{-1}\right)=(1 / 300-1 / 300.00255)=$ $2.83 \cdot 10^{-8} \mathrm{~K}^{-1}$. The residual process, i.e. entropy generation due to thermal contact resistances, amounts to $\left(\mathrm{d}_{\mathrm{i}} S / \mathrm{d} t\right)_{\text {res }}=\left(G_{\text {proc }}-G_{\text {noproc }}\right)(\delta Q / \mathrm{d} t)$, where $G_{\text {proc }}-G_{\text {noproc }}=1.864 \cdot 10^{-5}-2.83 \cdot 10^{-8}=$ $=1.861 \cdot 10^{-5} \mathrm{~K}^{-1}$. Statement (S.f) indicates that a heat flow across a thermal contact resistance cannot be associated with a true thermodynamic material property. [This is supported by the following analysis: the presence of air between the sheets - which normally occupy an effective volume fraction of around $1.5 \%-$ cannot through any continuum theory explain the sharp 
reduction of apparent thermal conductivity from 392 $\mathrm{W} / \mathrm{mK}$ to $0.59 \mathrm{~W} / \mathrm{mK}$. If this would be the case, a continuum theory would suggest a bulk thermal conductivity of the thermal contact resistance region according

$\lambda_{\text {apparent }}=\left(0.015 / \lambda_{\text {resistancelayer }}+0.985 / \lambda_{\mathrm{Cu}}\right)^{-1}=0.59$

, i.e. $\lambda_{\text {resistancelayer }}=0.0089 \mathrm{~W} / \mathrm{mK}$, arguably an

unreasonably low thermal conductivity (amounting to about $30 \%$ of the thermal conductivity of air).]

\section{A2. Chemical Reactions}

Let the parameter $\mathrm{d} \xi_{j} / \mathrm{d} t$ represent the instantaneous local flow rate of molecules of type $j$. Assume a total process, where carbon molecules $(C)$ and oxygen molecules $\left(\mathrm{O}_{2}\right)$ flow to the reaction, resulting in generation of carbon dioxide molecules $\left(\mathrm{CO}_{2}\right)$. Assume also a partial chemical reaction within this total process, where carbon molecules and oxygen molecules produce carbon monoxide $(\mathrm{CO})$. It is then of interest to analyze the residual chemical reaction that must occur in order to complete the total reaction. To proceed utilizing residual thermodynamics, one can assign entropy change relationships in terms of Eq. (1), clarified in corresponding real/total process and hypothetical/partial process comparisons corresponding to Eqs. (5)-(6), respectively. Identical thermodynamic flow vectors can be assumed (according to the fundamental hypothesis). One suitable thermodynamic flow vector connecting the absolute rates of molecular flows considered in the total and partial reactions is for instance $\mathrm{d} \mathbf{Y}=\left(\mathrm{d} \xi_{\mathrm{C}}, \mathrm{d} \xi_{\mathrm{O}_{2}}, \mathrm{~d} \xi_{\mathrm{CO}_{2}}, \mathrm{~d} \xi_{\mathrm{CO}}\right)$. The total entropy change can thus be expressed as $\mathrm{d}_{\mathrm{i}} S_{\text {tot }}=\left(2 \mu_{C} / T, 2 \mu_{O_{2}} / T,-2 \mu_{C O_{2}} / T, 0\right) \cdot \mathrm{d} \mathbf{Y}$ and the partial process entropy change as $\mathrm{d}_{\mathrm{i}} S_{\text {part }}=\left(2 \mu_{C} / T, \mu_{O_{2}} / T, 0,-2 \mu_{C O} / T\right) \cdot \mathrm{d} \mathbf{Y}$, where $\mu_{j}$ represents the chemical potentials. The residual chemical reaction is obtained by subtracting the partial- from the total entropy

$$
\begin{aligned}
& \mathrm{d}_{\mathrm{i}} S_{\text {res }}=\mathrm{d}_{\mathrm{i}} S_{\text {tot }}-\mathrm{d}_{\mathrm{i}} S_{\text {part }}=\left(\mathbf{F}_{\text {tot }}-\mathbf{F}_{\text {part }}\right) \cdot \mathrm{d} \mathbf{Y}= \\
& =\left(0, \mu_{\mathrm{O}_{2}} / T,-2 \mu_{\mathrm{CO}_{2}} / T, 2 \mu_{\mathrm{CO}} / T\right) \cdot \mathrm{d} \mathbf{Y} .
\end{aligned}
$$$$
\text { In effect, one }
$$
change:

$$
\mathrm{d}_{\mathrm{i}} S_{\mathrm{res}}=\left(\mu_{\mathrm{O}_{2}} / T\right) \mathrm{d} \xi_{\mathrm{O}_{2}}+\left(2 \mu_{\mathrm{CO}} / T\right) \mathrm{d} \xi_{\mathrm{CO}}-\left(2 \mu_{\mathrm{CO}_{2}} / T\right) \mathrm{d} \xi_{\mathrm{CO}_{2}} \text {. }
$$

Alternatively, practicing the traditional concept of chemical affinity represents a slightly different approach: $\mathrm{d}_{\mathrm{i}} S_{\text {tot }}=A_{\text {tot }} d \xi_{\text {tot }}=A_{3} d \xi_{3}=\mathrm{d}_{\mathrm{i}} S_{1}+\mathrm{d}_{\mathrm{i}} S_{2}$

$=A_{1} d \xi_{1}+A_{2} d \xi_{2}$. The rate of reactions for process 1 and 2 and the net reaction can then be expressed as $d \xi_{1}=d \xi_{\text {part }}=d N_{C} /-2=d N_{O_{2}} /-1=d N_{C O} / 2$, $d \xi_{2}=d \xi_{\text {res }}=d N_{O_{2}} /-1=d N_{C_{2}} / 2=d N_{C O} /-2$ and $\quad d \xi_{3}=d \xi_{\text {tot }}=d N_{C} /-2=d N_{\mathrm{O}_{2}} /-2=d N_{\mathrm{CO}_{2}} / 2$. The corresponding chemical affinities for the partial reactions can in turn be expressed as $A_{1}=\left(2 \mu_{C}+\mu_{O_{2}}-2 \mu_{C O}\right) / T$ and $A_{2}=\left(\mu_{\mathrm{O}_{2}}-2 \mu_{\mathrm{CO}_{2}}+2 \mu_{\mathrm{CO}}\right) / T$. Since the rates of the partial reactions are connected with each other and with the net reactions in a way that directly relates $d \xi_{1}=d \xi_{2}=d \xi_{3}, \quad$ one immediately obtains $A_{3}=A_{1}+A_{2}=\left(2 \mu_{C}+2 \mu_{O_{2}}-2 \mu_{C O_{2}}\right) / T$. An analysis utilizing the traditional chemical affinity expressions is useful, as it provides a scalar number representing an offset from chemical equilibrium. An identical residual thermodynamic expression in terms of chemical affinity can also be expressed: If setting $\mathrm{d} X=\mathrm{d} \xi_{1}=\mathrm{d} \xi_{2}=\mathrm{d} \xi_{3}$ and setting $G_{1}=A_{1}, \quad G_{2}=A_{2}, G_{3}=A_{3}$ according to the above, one still maintains the residual expression $\mathrm{d}_{\mathrm{i}} S_{\text {res }}=\mathrm{d}_{\mathrm{i}} S_{\text {tot }}-\mathrm{d}_{\mathrm{i}} S_{\text {part }}=\left(G_{3}-G_{1}\right) \mathrm{d} X \quad$ where the chemical reaction process yet remains within the category of a residual thermodynamic process. From statement (S.f) it can be concluded that a chemical reaction cannot be associated with any thermodynamic material property, i.e. a chemical affinity does not qualify as a thermodynamic material property.

\section{A3. Newtonian Fluid Dynamics}

Consider the case of a differential irreversible work loss of an incompressible, isothermal and stationary laminar flow caused by viscous stresses for a Newtonian fluid. To simplify, limit the case to a single-phase, inert Newtonian flow, also assuming no flow separation. The mechanisms of irreversible work loss due to fluid friction (viscous stresses) - utilizing Eq. (9) - can then be analyzed by comparing the corresponding cases of "process = viscous flow" and "no process = non-viscous flow", the latter commonly referred to as a "potential flow" in fluid dynamics. Studying the different parts in Eq. (9), it is immediately realized that term (a) is zero, due to the equation of continuity for incompressible flows, i.e. $\nabla \cdot \mathbf{U}=0, c f$. Navier-Stokes equations. Continuing with terms (b) and (d), one can note that these denote reversible processes, as they represent velocity times gradients of forces, effects that result in the acceleration of the fluid (whether it be non-viscous or viscous). Terms (b) and (d) representing reversible processes are true for both cases "process" and "no process", and hence do not relate to any irreversible work loss for a Newtonian fluid. What remains as an irreversible work is term (c), which, adjusting the sign in order to obtain an irreversible work loss, amounts to $\alpha\left(\begin{array}{l}=\bar{\tau}_{\text {proc }}-\tau_{\text {noproc }}\end{array}\right): \nabla \mathbf{U}$ . By definition, for a single-phase flow $\alpha=1$, and for a non-viscous flow $\bar{\tau}_{\text {noproc }}=\stackrel{=}{0}$. Finally, what remains of the work loss is $\tau_{\text {proc }}: \nabla \mathbf{U}$, an expression that does not qualify as a residual process (since the corresponding $\mathbf{F}_{\text {noproc }}=\mathbf{0}$ ).

Thus, to summarize, through Eq. (9) the irreversible work loss due to work of viscous stresses was identified. This is equal to $-\dot{w}_{\text {proc }}-(-\underbrace{\cdot \dot{w}_{\text {noproc }}}_{=0})=\bar{\tau}_{\text {proc }}: \nabla \mathbf{U}=\bar{\tau}: \nabla \mathbf{U}=\delta, \quad$ noting

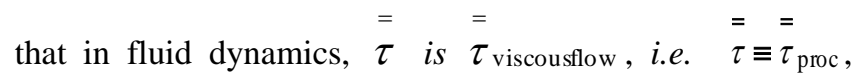
since $\bar{\tau}_{\text {noproc }}=\overline{0}$. Term $\delta$ is recognized as the viscous dissipation in fluid dynamics. The fact that viscous dissipation represents the irreversible work loss in a fluid 
element due to work of viscous stresses is a well-known result in fluid dynamics - although this has not previously been demonstrated in this way. Furthermore, according to statement (S.a) the process of viscous dissipation $\delta$ (and

tensor $\left.\bar{\tau}_{\text {viscousflow }}\right)$ can be directly associated with a thermodynamic material property through a linear phenomenological relation, in this case the kinematic viscosity of the Newtonian fluid.

\section{A4. Erosion Ripple Phenomenon - the Slip-Roll Mechanism}

One puzzling observation is the so-called erosion ripple phenomenon, which appears in some erosion experiments, $c f$. Figure A1, or in SIMS experiments. (The author has not found any source in the literature that details this mechanism. Regarding the ripple phenomenon observed in SIMS experiments, one recent reference in the literature suggests that a to date unknown structure with a wavelength of the order of 0.1 micrometers is somehow already present in the surface of the target specimen prior to the sputtering process, i.e. a material structure that has previously not been observed with any other surface analysis technique.)

It can be shown that a non-zero slip flow aligned with the target surface is a fundamental necessity, according to the $1^{\text {st }}$ law of thermodynamics, for ductile erosion (or abrasion) to occur, $c f$. (Gustavsson, submitted). In the following, the slip-roll mechanism is introduced.

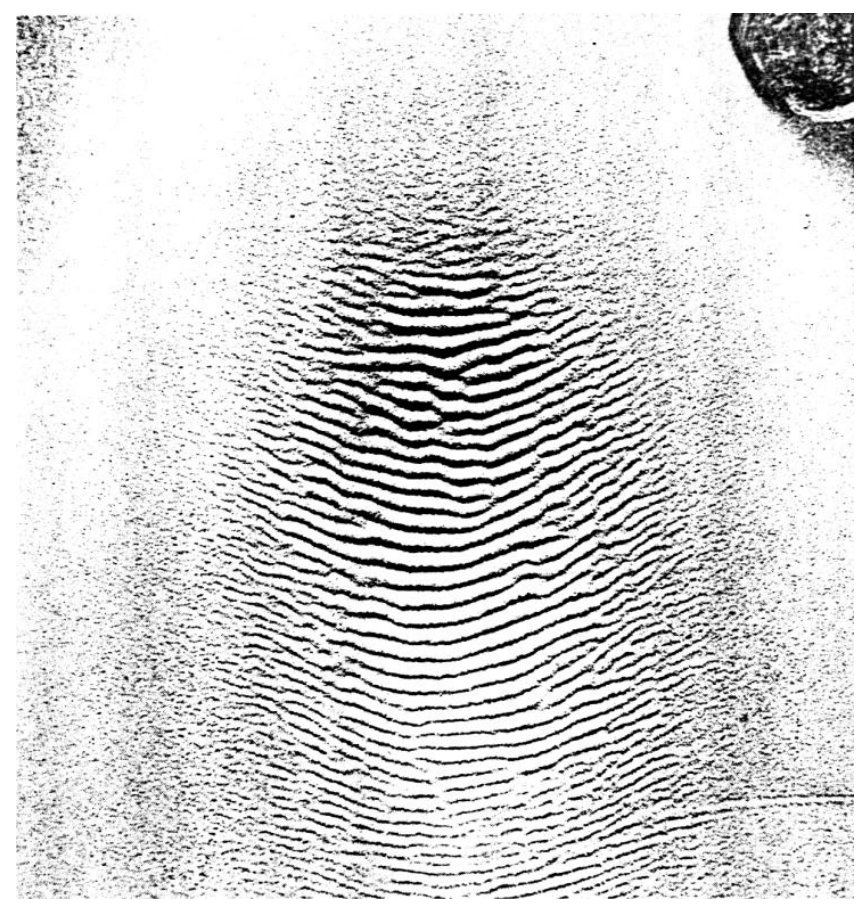

Figure A1. Erosion ripple pattern formed when commercially-pure Al is eroded by sand at a macroscopic jet impact angle of $30^{\circ}$, obtained by (Finnie \& Kabil, 1965). The particle slip flow is directed from the top to bottom on the photograph, with a local slip flow vector at $90^{\circ}$ to the ripple pattern. The wavelength approximately equals the particle diameter, or half the particle diameter. (Reproduced with permission.)

The slip-roll mechanism is presented in Figure A2. The erosion ripple wavelength is defined as the length $\lambda$, for which during the time interval $t_{1}$ the innermost particle slides at velocity $U_{\text {slip }}$ from position A to position $\mathrm{A}^{\prime}$, while simultaneously the second layer particle rolls from position B to B'. The second layer particle hence rolls over the first particle layer, and provides an additional residual fluctuation (in both effective thermodynamic force and flow, i.e. a dual-coupled fluctuation), for which the total entropy generation and net erosion rate will increase (in line with experimental observations by Finnie and Kabil (Finnie \& Kabil, 1965), who observed an increase in net total erosion rate, together with a more "turbulent" response of the exiting particles in connection with occurring erosion ripple phenomena).

It is shown in the fluid dynamics literature that a nonviscous slip-flow can trigger instability of any frequency. In the present case, a non-continuous situation is clearly at hand in which the continuum concept of viscosity does not apply. The most fundamental tone or frequency is the instability and frequency as triggered by the slip-roll mechanism.

A first estimation, based on the ideal geometry and ideal motion as indicated in Figure A2, can be made for macroscopic particles (sub-mm to $\mathrm{mm}$ range):

$$
U_{1}=U_{\text {slip }}=L \frac{\partial U}{\partial y} \approx L\left(U_{2}-U_{\text {slip }}\right) / d_{\mathrm{p}}
$$

(not to scale)

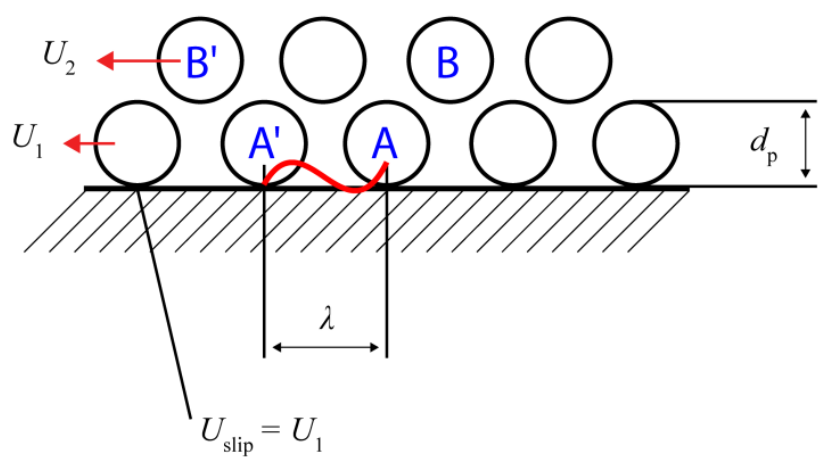

Figure A2. Slip-roll mechanism.

From the literature, according to kinetic theory ( $c f . e . g$. (Chapman \& Cowling, 1991) applied for macroscopic particles (Ding et al., 1992):

$$
L \cong \frac{\varphi d_{\mathrm{p}}}{6 \alpha_{\mathrm{p}} \sqrt{2}}
$$

The particles may be assumed to be perfectly round, i.e. as having a sphericity of $\varphi=1$ ( $c f$. note below on roundness requirement). From Eq. (A1a):

$U_{2} \approx U_{\text {slip }}\left(1+6 \sqrt{2} \alpha_{\mathrm{p}}\right)$

From Figure A2:

$s_{1}=U_{\text {slip }} \cdot t_{1}=\lambda$ 
It is also possible to approximate $S_{2}$ - accounting for the particle concentration (in the densest case in Figure A2 $\left.s_{2} \approx \lambda+1.5 d_{\mathrm{p}}\right)-$ as:

$s_{2} \approx \lambda+\frac{d_{\mathrm{p}}}{0.8 \alpha_{\mathrm{p}}^{1 / 3}}$

From these relations, the wavelength can be calculated as:

$\lambda \approx \frac{d_{\mathrm{p}}}{4.8 \sqrt{2} \alpha_{\mathrm{p}}^{4 / 3}} \cong \frac{d_{\mathrm{p}}}{6.8 \alpha_{\mathrm{p}}^{4 / 3}}$

For a particle volumetric concentration of $\alpha_{\mathrm{p}}=0.4$, which is slightly less than the particle concentration at minimum fluidization (Kuuni D \& Levenspiel O, 1991), one obtains $\lambda \approx 0.50 \cdot d_{\mathrm{p}}$. If the particle concentration at the surface decreases, one obtains longer wavelengths, for instance, at $\alpha_{\mathrm{p}}=0.3$ one obtains $\lambda \approx 0.73 \cdot d_{\mathrm{p}}$ and if $\alpha_{\mathrm{p}}=0.2$ one

obtains $\lambda \approx 1.26 \cdot d_{\mathrm{p}}$. This result provides magnitude agreement with experimental observations (where the wavelength is of the order of the particle diameter or half the particle diameter according to the literature, $c f$. e.g. (Finnie \& Kabil, 1965)). For a jet stream of impacting particles, the particle concentration is at a maximum in the center zone, and decreases towards the sides, $c f$. Figures 6-7 in (Gustavsson, 2002). The trend in wavelength vs. particle concentration, however, appears to be the opposite within the core erosion ripple zone, according to experimental results ( $c f$. Figure A1).

A refinement of the wavelength estimation in Eq. (A6a) can be made by incorporating an analysis of alterations in the geometrical assumptions, due to the presence of fluctuations. For instance, the amplitude of the dualcoupled fluctuations will alter the geometrical conditions in the following way: First, the parameter $L$ will significantly increase with increased fluctuations (independently of the particle concentration $\left.\alpha_{\mathrm{p}}\right)$, say $L \cong L_{\text {nofluctuation }} F_{1}\left(T_{\mathrm{g}}\right)$, where one can introduce a "granular temperature" $T_{\mathrm{g}}$ as a quantitative measure of the particle fluctuations. (In the field of multiphase flow, a granular temperature is often introduced. It is well known that an apparent increase in fluctuations or granular temperature will increase the apparent viscosity, and hence also the slip flow parameter $L$ in the vicinity of solid surfaces.) Secondly, the vertical distance [assumed to be $d_{\mathrm{p}}$ in Eq. (A1a)] in the approximation of the velocity derivative at the surface, will also increase with increased fluctuations. One can modify Eq. (A1a) into:

$U_{1} \approx F_{1}\left(T_{\mathrm{g}}\right) L_{\text {nofluctuations }}\left(U_{2}-U_{\text {slip }}\right) / F_{2}\left(T_{\mathrm{g}}\right) d_{\mathrm{p}}$

This gives a modified estimation of the wavelength:

$\lambda \approx \frac{F_{1}\left(T_{\mathrm{g}}\right)}{F_{2}\left(T_{\mathrm{g}}\right)} \cdot \frac{d_{\mathrm{p}}}{6.8 \alpha_{\mathrm{p}}^{4 / 3}}$

Within the erosion ripple zone of the target surface, increasing amplitude of the emerging ripple structure indicates continually increasing normal-direction fluctuations for particles in the vicinity of the target surface. From this observation, it is reasonable - and with support from multiphase flow theory - to suggest that the presence of fluctuations will increase the ratio $F_{1} / F_{2}$ with increasing granular temperature, i.e. approximately $F_{1}\left(T_{\mathrm{g}}\right) / F_{2}\left(T_{\mathrm{g}}\right) \propto T_{\mathrm{g}}{ }^{s}$, where $s>0$. Also, it is apparent that the time-averaged particle concentration $\alpha_{\mathrm{p}}=\alpha_{\mathrm{p}}\left(T_{\mathrm{g}}\right)$ in Eq. (A6b) will decrease with increased fluctuations, or granular temperature $T_{\mathrm{g}}$. Hence, the presence of fluctuations will according to Eq. (A6b) result in significantly increased wavelength, i.e. provide improved, and possibly excellent, agreement with experimental observations, $c f$. Figure A1. In addition, if a wavelength is known along a surface, together with an estimated particle concentration $\alpha_{\mathrm{p}}\left(T_{\mathrm{g}}\right)$ along the same surface, this will make it possible to calculate a variation of the ratio $F_{1}\left(T_{\mathrm{g}}\right) / F_{2}\left(T_{\mathrm{g}}\right)$ in Eq. (A6b). A variation of the ratio $F_{1}\left(T_{\mathrm{g}}\right) / F_{2}\left(T_{\mathrm{g}}\right)$ for the experiment in Figure A1 can be roughly estimated to be around 0.25 to 2 within the erosion ripple zone based on the above assumptions on variations in particle concentration $\alpha_{\mathrm{p}}=\alpha_{\mathrm{p}}\left(T_{\mathrm{g}}\right)$, since real particle concentration data along the surface in this experiment was not recorded by (Finnie \& Kabil, 1965). To conclude, timeaveraged particle concentration, particle diameter and amplitudes of fluctuations appear to locally control the resulting wavelength of the propagating wave (i.e. the erosion ripple structure).

The residual thermodynamic process of ductile erosion, with additional work-loss-induced fluctuations increasing the time-averaged residual entropy generation, generates a dissipative structure with a wavelength which is similar to, or less than the particle diameter. The continuum assumption is clearly not valid, as the dissipative structure wavelength is much smaller than the smallest scale possible to consider in a continuum assumption (for a multiphase particle flow). This is in accordance with statement (S.i), where residual thermodynamic processes also incorporate the possibility of collective behavior of many particles to render interactions on the sub-continuum scales. In addition, it is interesting to note that there is no material property included in the model for the erosion ripple wavelength in Eq. (A6b), associated with the target surface or the particles themselves, in line with statement (S.f). Furthermore, the specific flow speed of the macroscopic jet might have an influence on the granular temperature, which may in turn influence the detailed wavelength according to Eq. (A6b), whereas in the most simple slip-roll mechanism model in Eq. (A6a), the jet- or associated slip velocity does not influence the ripple wavelength. Again, as concluded earlier, it is geometry - not material properties - that primarily influences the resulting wavelength.

For smaller scales, similar ripple phenomena with regular wavelengths (typically 50-100 nm) can occur in connection with SIMS (secondary ion mass spectrometry) experiments. In this experiment ions bombard a target surface at a certain impact angle, which results in ductile erosion of the target surface. The eroded target surface material is collected and analyzed, providing a means for analyzing the material structure of the target surface. The ensuing erosion ripple wavelength can be analyzed in the 
same way through kinetic theory, modeling a slip-roll mechanism of the erosive ions. This analysis gives:

$$
\begin{aligned}
& U_{1} \approx L\left(U_{2}-U_{\text {slip }}\right) / L \\
& s_{2} \approx \lambda+L
\end{aligned}
$$

resulting in: $\lambda \approx L$

Thus the erosion-ripple wavelength observed in SIMS experiments corresponds to the mean-free path of the impacting ions in the vicinity of the target surface. The erosion ripple wavelength reported in the literature for SIMS experiments has hitherto not been associated with the mean-free path of impacting ions. However, apparently, the reported wavelengths are of the same order of magnitude as typical mean-free path numbers in gases at ambient conditions (i.e. at $1 \mathrm{~atm}$ ). The author has not found any published data on the mean-free path of impacting ions in the vicinity of the target surface in connection with SIMS experiments, so the proposed relation in Eq. (A6c) requires experimental confirmation.

Finally, returning to macroscopic scales, it has been reported in the literature that erosion rippling will not occur if macroscopic particles have sharp corners, or if the material surface is brittle. For brittle erosion, a different fundamental mechanism - not related to any slip mechanism - will erode the target surface (Gustavsson, submitted). In addition, it has been experimentally observed that it is easier to generate an erosion ripple dissipative structure, if the macroscopic impact angle of the jet of particles is small. (At jet impact angles close to zero the erosion ripple phenomena disappears (Finnie \& Kabil, 1965), for reasons explained in (Gustavsson, submitted).) Finally, the analysis is performed in 2D, since the fluidized flow behavior itself - in the vicinity of a target surface acts in a 2D manner. During the ductile erosion process the particle is assumed to be moving essentially only in the slip-flow direction, where a normal (with respect to the target surface plane) direction force is acting. One may speculate on instantaneous offsets from this assumption, which may well be the case. However, such fluctuations in the plane of the target surface, adjacent to the averaged slipflow direction, may reduce the normal-direction - with respect to the target surface plane - effective thermodynamic force to sub-threshold levels, where erosion does not occur.

\section{A5. Length of Paper Fibers Produced in a TMP Wood Refiner}

Figure A3 illustrates ductile- and brittle attrition (Gustavsson, submitted) occurring in a TMP wood chip refiner (Berg et al., 2003; Karlström,et al., 2008; Karlström et al., 2007). Attrition cannot occur continuously. Monitoring a particle trajectory when passing the refiner zone, which is of the order of 10 rotations and duration 1 second from entry (as a wood chip) to exit from the rim of the TMP refining zone (as a paper fiber), the "defibration" process (i.e. the attrition process as labeled by Pulp\&Paper scientists) can physically occur only at a finite number of positions, whereas most particle-bar passages occur without exceeding the threshold for ductile or brittle attrition (Gustavsson, submitted). For ductile attrition, where the particles are fairly evenly distributed across the TMP refining zone, $c f$. Figure A3, the mechanism controlling the dissipative structure is an inverse slip-roll mechanism. Ductile attrition occurs at a number of positions, evenly distributed. The bar-to-bar geometry, which can be associated with fluctuation of a regular wavelength, controls the resulting length of the paper fibers (i.e. inverse slip roll), which is a direct opposite behavior to the regular slip roll mechanism (where a given particle diameter or mean-free path generates a fluctuation of a certain wavelength).

For brittle attrition processes, particles tend to "flocculate" in the rotational direction, $c f$. Figure A3, a flocculation which - at certain discrete positions compresses the particle concentration to such an extent that the brittle attrition threshold is exceeded (i.e. through a spontaneous fluctuation, the effective thermodynamic force becomes greater than zero), and brittle attrition occurs predominantly in flocculation agglomeration zones. Hence, in this mode of particle defibration in a TMP wood chip refiner, the apparent behavior of particle motion within the TMP refiner is aptly characterized as more "chaotic", and resulting fiber lengths have a wide statistical distribution, cf. Figure A3.

It can be concluded that geometry - in the TMP refiner case a continual wearing-down and smoothening (or minor rounding) of the bars - has a great impact on the entire brittle and ductile attrition process (Gustavsson, submitted), as well as on associated respective dissipative structure behavior. The average fiber lengths, which influence final paper quality, strongly depend on the nature of these two modes of dissipative structures. 


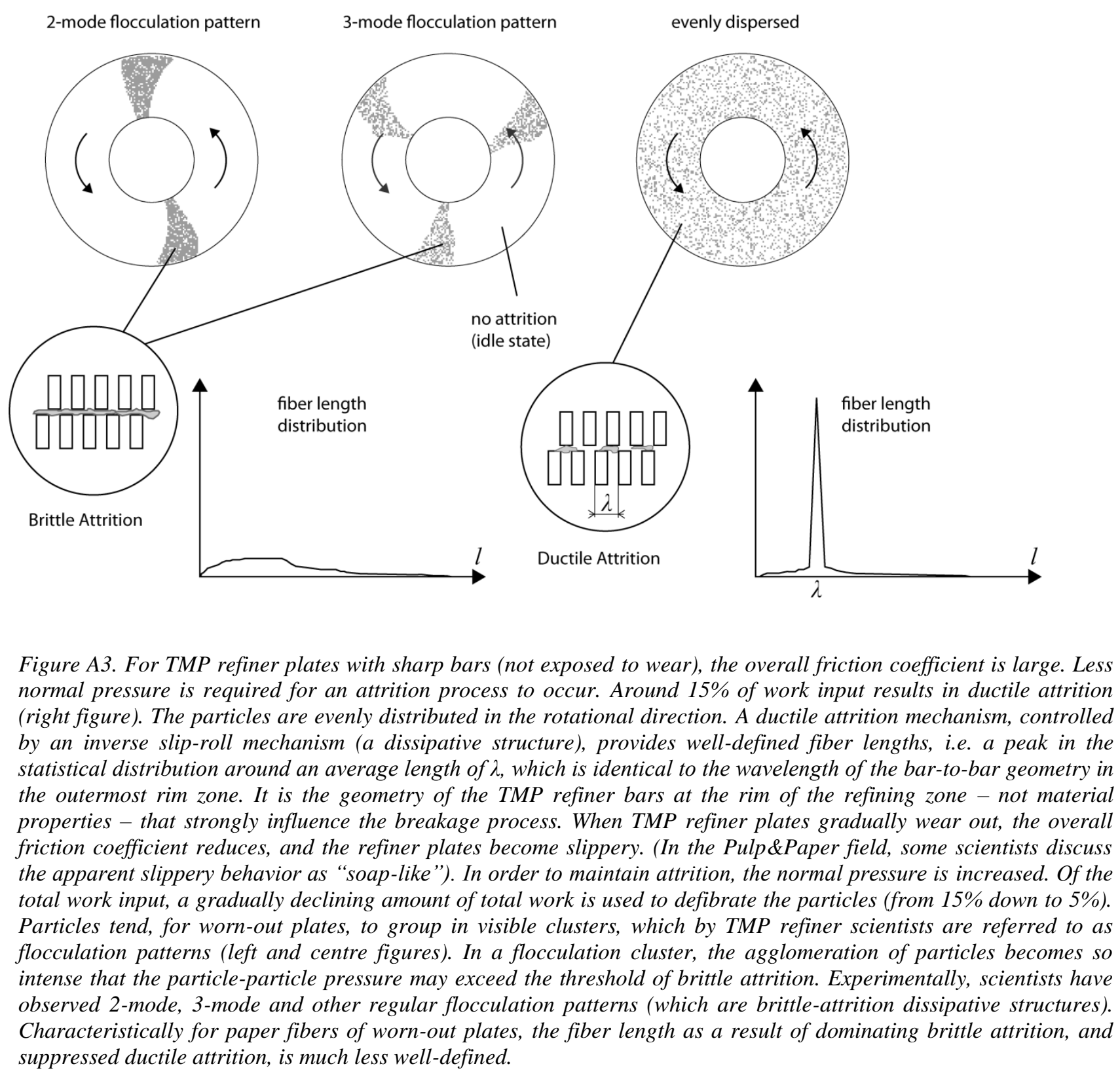

